\title{
Globally strictly convex cost functional for an inverse parabolic problem
}

\author{
Michael V. Klibanov* and Vladimir G. Kamburg ${ }^{\circ}$ \\ *Department of Mathematics and Statistics \\ University of North Carolina at Charlotte \\ Charlotte, NC 28223, USA \\ ${ }^{\circ}$ Department of Information and Computing Systems \\ Penza State University of Acrhitecture and Construction, \\ Penza 440028, Russian Federation \\ E-mails: mklibanv@uncc.edu and kamburg@rambler.ru
}

\begin{abstract}
A coefficient inverse problem for a parabolic equation is considered. Using a Carleman Weight Function, a globally strictly convex cost functional is constructed for this problem.
\end{abstract}

Keywords: coefficient inverse problem, Carleman estimate, global strict convexity 2010 Mathematics Subject Classification: 35R30.

\section{Introduction}

Both the most challenging and the most important question one needs to address when trying to solve numerically a Coefficient Inverse Problem (CIP) is: How to obtain a rigorously guaranteed good approximation for the exact solution without any advanced knowledge of a small neighborhood of this solution? We call a numerical method addressing this question globally convergent. The reason of the importance of this question is that conventional least squares cost functionals for CIPs are non-convex. Hence, they have many local minima and ravines. This makes numerical procedures, which use these functionals, unreliable. Indeed, to find a proper minimum, one should start the minimization process in a small neighborhood of the exact solution, i.e. one should start from a good first guess for the solution. However, if such a guess is known a priori, then why the exact solution is also not known a priori? In addition, such a neighborhood is very rarely available in practice. Therefore, it is important to develop globally convergent numerical methods for CIPs.

Currently there exist two types of such methods. Both for the case of the non overdetermined data, i.e. for the case of a single measurement event. The first type is based on constructions of globally strictly convex Tikhonov-like functionals. Carleman Weight Functions (CWFs) are the key to the global convexity. This method was initiated in 
works of Klibanov in 1997 [11, 12]; also see Klibanov and Timonov [13. Recently there is a renewed interest in Beilina and Klibanov [7] and Klibanov and Thánh [15] with some numerical studies in [15]. A different, although a similar approach, was carried out by Baudouin, De Buhan and Ervedoza [2]. The global convexity is understood as follows: Given a convex set $G$ of an arbitrary diameter $d$ in a certain Sobolev space, one can choose the parameter $\lambda_{0}=\lambda_{0}(d)>>1$ of the CWF such that for all $\lambda \geq \lambda_{0}$ that functional is strictly convex on $K$. Assume now that there exists a minimizer of that functional on the set $K$. Then the strict convexity guarantees convergence of the gradient method to this minimizer starting from any point of the set $K[7,15]$. This is the global convergence as in the above definition. Recall that in the conventional case the gradient method converges to a minimizer only if starting in a small neighborhood of that minimizer.

The second type of globally convergent numerical methods for CIPs is the method, which was initiated in the paper of Beilina and Klibanov [3] and was discussed since then in a number of follow up publications of these authors with coauthors. Results obtained before 2012 were summarized in the book [4]. In particular, this method was completely verified on experimental data, see, e.g., Chapter 5 in [4], as well as [5, 6, 17]. We also refer to a recent paper of Chow and Zou [9] for this method.

The method of this paper falls into category of the first type of globally convergent numerical methods. Using a CWF for the parabolic operator, we construct a globally strictly convex cost functional for a CIP for a general parabolic equation of the second order. Unlike this, Note that CIPs for hyperbolic PDEs were considered in [2, 7, 11, 15. Although a CIP for a parabolic PDE was considered in [12, the main difference of that work with the current paper is that in [12] a certain series was truncated, whereas truncation does not take place here.

In section 2 we state our inverse problem. The globally strictly convex cost functional for it is constructed in section 3. In section 4 we prove the main result of this paper, which is Theorem 1 .

\section{Statement of the problem}

Let $\Omega \subset \mathbb{R}^{3}$ be a bounded domain with a piecewise smooth boundary $\partial \Omega$. Let $T>0$ be an arbitrary number. Denote $\Omega_{T}=\Omega \times(-T, T)$. Let $\Gamma \subseteq \partial \Omega$ be a part of the boundary $\partial \Omega$ and let $\Gamma \in C^{2}$. Denote $\Gamma_{T}=\Gamma \times(-T, T)$. Consider the elliptic operator of the second order in $\Omega$,

$$
L u=\sum_{i, j=1}^{3} a_{i j}(x) u_{x_{i} x_{j}}+\sum_{i, j=1}^{3} b_{j}(x) u_{x_{j}}+c(x) u, x \in \Omega .
$$

Here

$$
\begin{gathered}
a_{i j}=a_{j i}, a_{i j} \in C^{1}(\bar{\Omega}) ; b_{j}, c \in C(\bar{\Omega}), \\
\mu_{1}|\xi|^{2} \leq \sum_{i, j=1}^{3} a_{i j}(x) \xi_{i} \xi_{j} \leq \mu_{2}|\xi|^{2}, \forall x \in \bar{\Omega}, \forall \xi \in \mathbb{R}^{n},
\end{gathered}
$$

where $\mu_{1}, \mu_{2}=$ const. $>0, \mu_{1} \leq \mu_{2}$. Let the function $u \in C^{4,2}\left(\bar{\Omega}_{T}\right)$ satisfies the following conditions

$$
\begin{aligned}
& u_{t}=L u \text { in } \Omega_{T}, \\
& u(x, 0)=f(x) .
\end{aligned}
$$


We assume here that equation (2.4) is valid not only for $t>0$, but for $t<0$ as well. This is because the Bukhgeim-Klibanov method [8] does not work for the case when a parabolic equation is valid only for $t>0$ [4, 13, 14]. Our interest is in the inverse problem which we now formulate.

Coefficient Inverse Problem (CIP). Assume that the coefficient $c(x)$ in (2.1) is unknown for $x \in \Omega$. On the other hand, assume that the following functions $g_{1}(x, t), g_{2}(x, t)$ are known,

$$
\left.u\right|_{\Gamma_{T}}=g_{1}(x, t),\left.\partial_{n} u\right|_{\Gamma_{T}}=g_{2}(x, t) .
$$

Determine $c(x)$ for $x \in \Omega$.

Assume that in (2.5)

$$
f(x) \geq 2 b=\text { const. }>0 \text { in } \bar{\Omega}^{d} .
$$

Then uniqueness of this CIP follows immediately from Theorem 1.10.7 of [4] as well as from Theorem 3.3.2 of [13] and Theorem 3.4 of [14]. Note that a particular case of the data (2.6) is the case of backscattering data. The principal parts of the operators $L$ and $\partial_{t}-L$ we denote as $L_{0}, P_{0}$,

$$
L_{0} u=\sum_{i, j=1}^{3} a_{i j}(x) u_{x_{i} x_{j}}, P_{0} u=u_{t}-L_{0} u .
$$

\section{The cost functional}

To construct a globally convergent numerical method for our CIP, we construct in this section a globally strictly convex cost functional. Below $x=\left(x_{1}, \bar{x}\right)$, where $\bar{x}=\left(x_{2}, x_{3}\right)$. Without any loss of generality we can assume that $\Gamma=\left\{x: x_{1}=p(\bar{x}), p \in C^{2}(|\bar{x}| \leq \sqrt{d})\right\}$ for a number $d \in(0,1)$. Therefore, changing variables as $\left(x_{1}, \bar{x}\right) \Leftrightarrow\left(x_{1}^{\prime}, \bar{x}\right)$, where $x_{1}^{\prime}:=x_{1}-p(\bar{x})$, and using the same notations as before, for brevity, we conclude that we can assume that

$$
\Gamma=\{x: x=(0, \bar{x}),|\bar{x}|<\sqrt{d}\} .
$$

Denote

$$
\Omega^{d}=\left\{x: x_{1}>0,|\bar{x}|<\sqrt{d}\right\}
$$

and assume below that $\Omega^{d} \subset \Omega$. Then $\Gamma \subset \partial \Omega^{d}$. Below we determine the unknown coefficient $c(x)$ only in a subdomain of the domain $\Omega^{d}$.

We now formulate the Carleman estimate for the operator $P_{0}=\partial_{t}-L_{0}$. Let $\lambda>1$ and $\nu>1$ be two large parameters, which we define later. Consider an arbitrary number $a \in(0, d)$. Consider functions $\psi(x, t), \varphi_{\lambda}(x, t)$ defined as

$$
\psi(x, t)=x_{1}+|\bar{x}|^{2}+\frac{t^{2}}{T^{2}}+a, \varphi_{\lambda}(x, t)=\exp \left(\lambda \psi^{-\nu}\right) .
$$

Consider the following sets

$$
\begin{gathered}
G_{a, d}=\left\{(x, t): x_{1}>0, x_{1}+|\bar{x}|^{2}+\frac{t^{2}}{T^{2}}+a<d\right\}, \\
G_{a, d}^{0}=G_{a, d} \cap\{t=0\},
\end{gathered}
$$




$$
\begin{gathered}
\xi_{a, d}=\left\{(x, t): x_{1}>0, x_{1}+|\bar{x}|^{2}+\frac{t^{2}}{T^{2}}+a=d\right\}, \\
\Gamma_{a, d, T}=\left\{(x, t): x_{1}=0,|\bar{x}|^{2}+\frac{t^{2}}{T^{2}}<d-a\right\} \subset \Gamma_{T}, \\
\partial G_{a . d}=\xi_{a, d} \cup \Gamma_{a, d, T}, \\
G_{a, d-\varepsilon}=\left\{(x, t): x_{1}>0, x_{1}+|\bar{x}|^{2}+\frac{t^{2}}{T^{2}}+a<d-\varepsilon\right\},
\end{gathered}
$$

where $\varepsilon \in(0, d-a)$ is a sufficiently small number. Clearly $G_{a, d-\varepsilon} \subset G_{a, d}$. Since $d \in(0,1)$, then $G_{a, d} \subset \Omega_{T}, G_{a, d}^{0} \subset \Omega^{d}$ Also, $\xi_{a}$ is the level surface of both functions $\psi, \varphi_{\lambda}$. Note that $\xi_{a, d}$ is the level surface of the function $\varphi_{\lambda}$,

$$
\min _{\bar{G}_{a, d}} \varphi_{\lambda}^{2}=\left.\varphi_{\lambda}^{2}\right|_{\xi_{a, d}}=\exp \left[2 \lambda d^{-\nu}\right] .
$$

Lemma 1 follows immediately from Lemma 3 of $\S 1$ of chapter 4 of the book of Lavrentiev, Romanov and Shishatskii [16].

Lemma 1 (Carleman estimate). There exist sufficiently large numbers $\nu_{0}, \lambda_{0}>1$,

$$
\nu_{0}=\nu_{0}\left(a, d, \mu_{1}, \mu_{2}, \max _{i, j}\left\|a_{i, j}\right\|_{C^{1}\left(\bar{\Omega}^{d}\right)}, T\right), \lambda_{0}=\lambda_{0}\left(a, d, \mu_{1}, \mu_{2}, \max _{i, j}\left\|a_{i, j}\right\|_{C^{1}\left(\bar{\Omega}^{d}\right)}, T\right)
$$

depending only on listed parameters and a sufficiently large absolute constant $\lambda_{0}>1$ such that for all $\nu \geq \nu_{0}, \lambda \geq \lambda_{0}$ and for all functions $u \in C^{2,1}\left(\bar{G}_{a, d}\right)$ the following pointwise Carleman estimate is valid for all $(x, t) \in G_{a, d}$

$$
\begin{aligned}
\left(P_{0} u\right)^{2} \varphi_{\lambda}^{2} & \geq C \lambda|\nabla u|^{2} \varphi_{\lambda}^{2}+C \lambda^{3} u^{2} \varphi_{\lambda}^{2}+\operatorname{div} U+V_{t} \\
|U|,|V| & \leq C \lambda^{3}\left[(\nabla u)^{2}+u_{t}^{2}+u^{2}\right] \varphi_{\lambda}^{2}
\end{aligned}
$$

where the constant $C=C\left(n, \max _{i, j}\left\|a_{i, j}\right\|_{C^{1}\left(\bar{\Omega}^{d}\right)}\right)>0$ depends only on listed parameters.

Denote

$$
L_{c} u=L u-c(x) u=\sum_{i, j=1}^{3} a_{i j}(x) u_{x_{i} x_{j}}+\sum_{i, j=1}^{3} b_{j}(x) u_{x_{j}}
$$

Since the function $u \in C^{4,2}\left(\bar{\Omega}_{T}\right)$, then (2.7) implies that $u(x, t) \geq b>0$ in $\bar{G}_{a, d}$ for sufficiently small $T$. Hence, we can consider the function $v(x, t)=\ln u(x, t)$. Substituting $u=e^{v}$ in (2.4) and (2.5), we obtain

$$
\begin{gathered}
v_{t}=L_{c} v+\sum_{i, j=1}^{n} a_{i, j}(x) v_{x_{i}} v_{x_{j}}+c(x) \text { in } \bar{G}_{a, d}, \\
v(x, 0)=\ln f(x) .
\end{gathered}
$$

Let $w(x, t)=v_{t}(x, t)$. Differentiate (3.5) with respect to $t$ and use (3.6). We obtain the following nonlinear integral differential equation with respect to the function $w$ in the domain in $\bar{G}_{a, d}$

$$
w_{t}=L_{c} w+
$$




$$
\begin{aligned}
& +\sum_{i, j=1}^{n} a_{i, j}(x) w_{x_{i}}\left((\ln f)_{x_{j}}+\int_{0}^{t} w_{x_{j}}(x, \tau) d \tau\right) \\
& +\sum_{i, j=1}^{n} a_{i, j}(x) w_{x_{j}}\left((\ln f)_{x_{i}}+\int_{0}^{t} w_{x_{i}}(x, \tau) d \tau\right) .
\end{aligned}
$$

In addition, conditions (2.6) imply that

$$
\left.w\right|_{\Gamma_{a, d, T}}=\widetilde{g}_{1}(x, t),\left.\partial_{x_{1}} w\right|_{\Gamma_{a, d, T}}=\widetilde{g}_{2}(x, t),
$$

where

$$
\widetilde{g}_{1}(x, t)=\partial_{t} \ln g_{1}(x, t), \widetilde{g}_{2}(x, t)=\frac{g_{2 t}}{g_{1}}-\frac{g_{1 t} g_{2}}{g_{1}^{2}} .
$$

Since functions $g_{1}, g_{2}$ are the data for the inverse problem, then they naturally contain noise. Even though the differentiation of a noisy function is an ill-posed problem, it can be handled by a number of well known regularization methods, see, e.g. Aristov [1].

Thus, we have obtained the nonlinear integral differential equation (3.7) with the lateral Cauchy data (3.8). If we find the solution of this problem, then backwards calculations will deliver us the target coefficient $c(x)$ for $x \in G_{a, d}^{0}$. Hence, we focus below on the solution of the problem (3.7), (3.8). Denote $\widetilde{L} w$ the right hand side of (3.7), plus the term $-w_{t}$,

$$
\begin{gathered}
\widetilde{L} w=-w_{t}+L_{c} w+ \\
+\sum_{i, j=1}^{n} a_{i, j}(x) w_{x_{i}}\left((\ln f)_{x_{j}}+\int_{0}^{t} w_{x_{j}}(x, \tau) d \tau\right) \\
+\sum_{i, j=1}^{n} a_{i, j}(x) w_{x_{j}}\left((\ln f)_{x_{i}}+\int_{0}^{t} w_{x_{i}}(x, \tau) d \tau\right) .
\end{gathered}
$$

Our weighted Tikhonov-like cost functional is

$$
J_{\lambda, \alpha}(w)=\exp \left(-3 \lambda d^{-\nu}\right) \int_{G_{a, d}}(\widetilde{L} w)^{2} \varphi_{\lambda}^{2} d x d t+\alpha\|w\|_{H^{4}\left(G_{a, d}\right)}^{2},
$$

where $\alpha \in(0,1)$ is the regularization parameter. We use the multiplier $\exp \left(-3 \lambda d^{-\nu}\right)$ to ensure that we can indeed choose $\alpha \in(0,1)$, see Theorem 1 . We use the $H^{4}\left(G_{a, d}\right)$ - norm here since we need in our proof $w \in C^{1}\left(\bar{G}_{a, d}\right) \cap H^{2}\left(G_{a, d}\right)$, and the embedding theorem guarantees that

$$
H^{4}\left(G_{a, d}\right) \subset C^{1}\left(\bar{G}_{a, d}\right),\|u\|_{C^{1}\left(\bar{G}_{a, d}\right)} \leq C_{1}\|u\|_{H^{4}\left(G_{a, d}\right)}, \forall u \in H^{4}\left(G_{a, d}\right),
$$

where $C_{1}=C_{1}\left(G_{a, d}\right)>0$ is a generic constant depending only on the domain $G_{a, d}$. Thus, we consider below the following problem.

Minimization Problem. Minimize the functional $J_{\lambda, \alpha}(w)$ in (3.10), subject to the lateral Cauchy data (3.8).

Let $R>0$ be an arbitrary number. Consider the set $B(R) \subset H^{4}\left(G_{a, d}\right)$,

$$
B(R)=
$$




$$
\left\{w \in H^{4}\left(G_{a, d}\right):\|w\|_{H^{4}\left(G_{a, d}\right)}<R, w \text { satisfies boundary conditions (3.8) }\right\} .
$$

Introduce the space $H_{0}^{4}\left(G_{a, d}\right)$ as

$$
H_{0}^{4}\left(G_{a, d}\right)=\left\{u \in H^{4}\left(G_{a, d}\right):\left.u\right|_{\Gamma_{a, d, T}}=\left.\partial_{x_{1}} u\right|_{\Gamma_{a, d, T}}=0\right\} .
$$

Theorem 1. For all numbers $\lambda, \nu, \alpha>0$ and for all functions $w \in B(R)$ there exists the Frechét derivative $J_{\lambda, \alpha}^{\prime}(w) \in H_{0}^{4}\left(G_{a, d}\right)$ of the functional $J_{\lambda, \alpha}$ at the point $w$. Let $\nu=\nu_{0}$ be the sufficiently large number of Lemma 1 and $b>0$ be the number in (2.7). There exists a sufficiently large number $\lambda_{1}$,

$$
\lambda_{1}=\lambda_{1}\left(a, d, \mu_{1}, \mu_{2}, \max _{i, j}\left\|a_{i, j}\right\|_{C^{1}\left(\bar{\Omega}^{d}\right)}, T, b, R,\|\nabla f\|_{C\left(\bar{G}_{a, d}^{0}\right)}\right) \geq \lambda_{0}>1
$$

depending on listed parameters such that if the regularization parameter

$\alpha \in\left(\exp \left(-\lambda /\left(2 d^{\nu}\right)\right), 1\right)$, then the functional $J_{\lambda, \alpha}(w)$ is strictly convex on the set $B(R)$ for all $\lambda \geq \lambda_{1}$. More precisely,

$$
\begin{gathered}
J_{\lambda, \alpha}\left(w_{2}\right)-J_{\lambda, \alpha}\left(w_{1}\right)-J_{\lambda, \alpha}^{\prime}\left(w_{1}\right)\left(w_{2}-w_{1}\right) \geq \\
C_{1} \exp (2 \lambda q) \int_{G_{a+\varepsilon, d}}\left[\left(\nabla w_{2}-\nabla w_{1}\right)^{2}+\left(w_{2}-w_{1}\right)^{2}\right] d x d t+\frac{\alpha}{2}\left\|w_{2}-w_{1}\right\|_{H^{4}\left(G_{a, d}\right)}^{2}, \\
\forall w_{1}, w_{2} \in B(R), \forall \lambda \geq \lambda_{1} .
\end{gathered}
$$

where $q=(d-\varepsilon)^{-\nu_{0}}\left[1-3(d-\varepsilon)^{\nu_{0}} /(2 d)^{\nu_{0}}\right]>0$ and the constant $C_{1}>0$ depends on the same parameters as those in (3.13).

Note that we can require $\alpha \in\left(\exp \left(-\lambda /\left(2 d^{\nu}\right)\right), 1\right)$, since $\exp \left(-\lambda /\left(2 d^{\nu}\right)\right)<<1$ for sufficiently large $\lambda$. This theorem is the main result of our paper. Theorem 1 enables one to prove the convergence of the gradient method, which can start at any point of the set $B(R)$. Since there are no restrictions on the diameter $2 R$ of this set, then this is the global convergence as defined in Introduction. The stability with respect to the noise in the data (3.8) can also be established using this theorem. These two latter results can be derived from Theorem 1 in the same manner as similar results are derived from global strict convexity theorems in [7, 15]. Hence, we do not describe these results here. Below we focus on the proof of Theorem 1 and assume that its conditions are satisfied. Below $C_{1}>0$ denotes different constants depending on the same parameters as ones listed in (3.13).

\section{Proof of Theorem 1}

Let $w_{1}, w_{2} \in B(R)$ be two arbitrary functions. Denote $h=w_{2}-w_{1}$. Then

$$
h \in H_{0}^{4}\left(G_{a, d}\right),\|h\|_{H^{4}\left(G_{a, d}\right)} \leq 2 R .
$$

Let $A=\left(\widetilde{L}\left(w_{1}+h\right)\right)^{2}-\left(\widetilde{L}\left(w_{1}\right)\right)^{2}$. First, we single out the linear part of this expression with respect to $h$. Using (2.1) and (3.9), we obtain

$$
\widetilde{L}\left(w_{1}+h\right)=\widetilde{L}\left(w_{1}\right)+L_{c}(h)-h_{t}
$$




$$
\begin{aligned}
& +\sum_{i, j=1}^{3} a_{i, j}(x) h_{x_{i}}\left((\ln f)_{x_{j}}+\int_{0}^{t} w_{1 x_{j}}(x, \tau) d \tau\right)+ \\
& +\sum_{i, j=1}^{3} a_{i, j}(x) h_{x_{j}}\left((\ln f)_{x_{i}}+\int_{0}^{t} w_{1 x_{i}}(x, \tau) d \tau\right)+ \\
& +\sum_{i, j=1}^{3} a_{i, j}(x)\left[w_{1 x_{i}} \int_{0}^{t} h_{x_{j}}(x, \tau) d \tau+w_{1 x_{j}} \int_{0}^{t} h_{x_{i}}(x, \tau) d \tau\right] .
\end{aligned}
$$

Hence,

$$
\begin{array}{r}
A=2 \widetilde{L}\left(w_{1}\right)\left[L(h)-h_{t}+\sum_{i, j=1}^{3} a_{i, j}(x) h_{x_{i}}\left((\ln f)_{x_{j}}+\int_{0}^{t} w_{1 x_{j}}(x, \tau) d \tau\right)\right] \\
+2 \widetilde{L}\left(w_{1}\right) \sum_{i, j=1}^{3} a_{i, j}(x) h_{x_{j}}\left((\ln f)_{x_{i}}+\int_{0}^{t} w_{1 x_{i}}(x, \tau) d \tau\right) \\
+2 \widetilde{L}\left(w_{1}\right) \sum_{i, j=1}^{3} a_{i, j}(x)\left[w_{1 x_{i}} \int_{0}^{t} h_{x_{j}}(x, \tau) d \tau+w_{1 x_{j}} \int_{0}^{t} h_{x_{i}}(x, \tau) d \tau\right]+S^{2}\left(h, w_{1}\right),
\end{array}
$$

where

$$
\begin{gathered}
S\left(h, w_{1}\right)=L(h)-h_{t} \\
+\sum_{i, j=1}^{3} a_{i, j}(x) h_{x_{i}}\left((\ln f)_{x_{j}}+\int_{0}^{t} w_{1 x_{j}}(x, \tau) d \tau\right) \\
+\sum_{i, j=1}^{3} a_{i, j}(x) h_{x_{j}}\left((\ln f)_{x_{i}}+\int_{0}^{t} w_{1 x_{i}}(x, \tau) d \tau\right) \\
+\sum_{i, j=1}^{3} a_{i, j}(x)\left[w_{1 x_{i}} \int_{0}^{t} h_{x_{j}}(x, \tau) d \tau+w_{1 x_{j}} \int_{0}^{t} h_{x_{i}}(x, \tau) d \tau\right] .
\end{gathered}
$$

The expression $D\left(w_{1}, h\right)=A-S^{2}\left(h, w_{1}\right)$ is linear with respect to $h$. Hence, consider the functional

$$
Q_{\lambda, \alpha}(h)=\exp \left(-3 \lambda d^{-\nu}\right) \int_{G_{a, d}} D\left(w_{1}, h\right) \varphi_{\lambda}^{2} d x d t+2 \alpha\left[h, w_{1}\right],
$$

where [,] is the scalar product in $H^{4}\left(G_{a, d}\right)$. This is a linear bounded functional acting from $H_{0}^{4}\left(G_{a, d}\right)$ into $\mathbb{R}$. Hence, by Riesz theorem there exists unique element $U_{\lambda, \alpha}\left(w_{1}\right) \in$ $H_{0}^{4}\left(G_{a, d}\right)$ such that $Q_{\lambda, \alpha}(h)=\left[U_{\lambda, \alpha}\left(w_{1}\right), h\right]$. Furthermore, the norm $\left\|U_{\lambda, \alpha}\right\|_{H^{4}\left(G_{a, d}\right)}$ equals to the norm of the functional $Q_{\lambda, \alpha}$. Hence, we have proven the existence of the Frechét derivative $J_{\lambda, \alpha}^{\prime}\left(w_{1}\right)=U_{\lambda, \alpha}\left(w_{1}\right) \in H_{0}^{4}\left(G_{a, d}\right)$ of the functional $J_{\lambda, \alpha}$ and

$$
J_{\lambda, \alpha}^{\prime}\left(w_{1}\right)(h)=\exp \left(-3 \lambda d^{-\nu}\right) \int_{G_{a, d}} B\left(w_{1}, h\right) \varphi_{\lambda}^{2} d x d t+2 \alpha\left[h, w_{1}\right], \forall h \in H_{0}^{4}\left(G_{a, d}\right) .
$$


Hence,

$$
\begin{gathered}
J_{\lambda, \alpha}\left(w_{1}+h\right)-J_{\lambda, \alpha}\left(w_{1}\right)-J_{\lambda, \alpha}^{\prime}\left(w_{1}\right)(h) \\
=\exp \left(-3 \lambda d^{-\nu}\right) \int_{G_{a, d}} S^{2}\left(h, w_{1}\right) \varphi_{\lambda}^{2} d x d t+\alpha\|h\|_{H^{4}\left(G_{a, d}\right)}^{2},
\end{gathered}
$$

where $S\left(h, w_{1}\right)$ is given in (4.2).

We now focus on the estimate from the below of the integral in (4.3). Because of Lemma 1, we single out the term with $\left(h_{t}-L(h)\right)^{2}$. Using (2.2), (2.7), (3.9) and the Cauchy-Schwarz inequality, we obtain

$$
\begin{gathered}
S^{2}\left(h, w_{1}\right) \geq \frac{1}{2}\left(h_{t}-L(h)\right)^{2}-C_{1}(\nabla h)^{2}-C_{1}\left(\int_{0}^{t}|\nabla h(x, \tau)|^{2} d \tau\right) \\
\geq \frac{1}{3}\left(P_{0}(h)\right)^{2}-C_{1}(\nabla h)^{2}-C_{1}\left(\int_{0}^{t}|\nabla h(x, \tau)| d \tau\right)^{2},
\end{gathered}
$$

where the operator $P_{0}=\partial_{t}-L_{0}$ was defined in (2.8). It follows from Lemma 1.10.3 of [4] that

$$
\int_{G_{a, d}}\left(\int_{0}^{t}|\nabla h(x, \tau)| d \tau\right)^{2} \varphi_{\lambda}^{2} d x d t \leq \frac{C_{1}}{\lambda} \int_{G_{a, d}}(\nabla h)^{2} \varphi_{\lambda}^{2} d x d t .
$$

Hence, using Lemma 1, (3.3), (3.4), (4.1), (4.4) and (4.5), we obtain for sufficiently large $\lambda \geq \lambda_{1}$

$$
\begin{gathered}
\exp \left(-3 \lambda d^{-\nu}\right) \int_{G_{a, d}} S^{2}\left(h, w_{1}\right) \varphi_{\lambda}^{2} d x d t \geq C_{1} \lambda \exp \left(-3 \lambda d^{-\nu}\right) \int_{G_{a, d}}\left[(\nabla h)^{2}+h^{2}\right] \varphi_{\lambda}^{2} d x d t \\
-C_{1} \exp \left(-3 \lambda d^{-\nu}\right) \int_{G_{a, d}}(\nabla h)^{2} \varphi_{\lambda}^{2} d x d t-C_{1} \exp \left(-\lambda d^{-\nu}\right) \int_{\xi_{a, d}}\left[h_{t}^{2}+(\nabla h)^{2}+h^{2}\right] d \sigma \\
\geq C_{1} \lambda \exp \left(-3 \lambda d^{-\nu}\right) \int_{G_{a, d}}\left[(\nabla h)^{2}+h^{2}\right] \varphi_{\lambda}^{2} d x d t-C_{1} \exp \left(-\lambda d^{-\nu}\right) \int_{\xi_{a, d}}\left[h_{t}^{2}+(\nabla h)^{2}+h^{2}\right] d \sigma \\
\geq C_{1} \exp (2 \lambda q) \int_{G_{a, d-\varepsilon}}\left[(\nabla h)^{2}+h^{2}\right] d x d t-C_{1} \exp \left(-\lambda d^{-\nu}\right) \int_{\xi_{a, d}}\left[h_{t}^{2}+(\nabla h)^{2}+h^{2}\right] d \sigma .
\end{gathered}
$$

Thus, we have established that

$$
\begin{gathered}
\exp \left(-3 \lambda d^{-\nu}\right) \int_{G_{a, d}} S^{2}\left(h, w_{1}\right) \varphi_{\lambda}^{2} d x d t \\
\geq C_{1} \exp (2 \lambda q) \int_{G_{a, d-\varepsilon}}\left[(\nabla h)^{2}+h^{2}\right] d x d t-C_{1} \exp \left(-\lambda d^{-\nu}\right) \int_{\xi_{a, d}}\left[h_{t}^{2}+(\nabla h)^{2}+h^{2}\right] d \sigma .
\end{gathered}
$$


Next, since $\alpha \in\left(\exp \left(-\lambda /\left(2 d^{\nu}\right)\right), 1\right)$, then

$$
-C_{1} \exp \left(-\lambda d^{-\nu}\right) \int_{\xi_{a, d}}\left[h_{t}^{2}+(\nabla h)^{2}+h^{2}\right] d \sigma \geq-\frac{\alpha}{2}\|h\|_{H^{4}\left(G_{a, d}\right)}^{2} .
$$

Combining (4.3) with (4.6) and (4.7), we obtain the target estimate (3.14).

\section{References}

[1] Arestov VV. Approximation of unbounded operators by bounded operators and related extremal problems. Russian Mathematical Surveys 1996; 51: 1093-1126.

[2] Baudouin L, De Buhan M, Ervedoza S. Global Carleman estimates for waves and applications. Communications in Partial Differential Equations 2013; 38: 823-859.

[3] Beilina L, Klibanov MV. A globally convergent numerical method for a coefficient inverse problem. SIAM Journal on Scientific Computing 2008; 31: 478-509.

[4] Beilina L, Klibanov MV. Approximate Global Convergence and Adaptivity for Coefficient Inverse Problems. Springer: New York, 2012.

[5] Beilina L, Thành NT, Klibanov MV, Malmberg JB. Globally convergent and adaptive finite element methods in imaging of buried objects from experimental backscattering radar measurements. Journal on Computational and Applied Mathematics, DOI: 10.1016/j.cam.2014.11.055.

[6] Beilina L, Thánh NT, Klibanov MV, Fiddy MA. Reconstruction from blind experimental data for an inverse problem for a hyperbolic equation. Inverse Problems 2014; 30: 025002 .

[7] Beilina L, Klibanov MV. Globally strongly convex cost functional for a coefficient inverse problem. Nonlinear Analysis: Real World Applications 2015; 22: 272-288.

[8] Bukhgeim AL, Klibanov MV. Uniqueness in the large of a class of multidimensional inverse problems. Soviet Mathematics Doklady 1981; 17: 244-247.

[9] Chow YT, Zou J. A numerical method for reconstructing the coefficient in a wave equation. Numerical Methods for Partial Differential Equations 2015; 31: 289-307.

[10] Isakov V. Inverse Problems for Partial Differential Equations. Second Edition. Springer: New York, 2006.

[11] Klibanov MV. Global convexity in a three-dimensional inverse acoustic problem. SIAM Journal on Mathematical Analysis 1997; 28: 1371-1388.

[12] Klibanov MV. Global convexity in diffusion tomography. Nonlinear World 1997; 4: 247-265.

[13] Klibanov MV, Timonov A. Carleman Estimates for Coefficient Inverse Problems and Numerical Applications. VSP: Utrecht, 2004. 
[14] Klibanov MV. Carleman estimates for global uniqueness, stability and numerical methods for coefficient inverse problems. Journal of Inverse and Ill-Posed Problems 2013; 21: 477-560.

[15] M.V. Klibanov and N.T. Thánh, Recovering of dielectric constants of explosives via a globally strictly convex cost functional, arxiv 1408.0583v1 [math-ph], August 4, 2014. SIAM Journal on Applied Mathematics. Accepted for publication.

[16] Lavrentiev MM, Romanov VG, Shishatskii SP. Ill-Posed Problems of Mathematical Physics and Analysis. AMS: Providence, RI, 1986.

[17] Thánh NT, Beilina L, Klibanov MV, Fiddy MA. Reconstruction of the refractive index from experimental backscattering data using a globally convergent inverse method. SIAM Journal on Scientific Computing 2014; 36: B273-B293. 